\title{
Energy Efficiency Analysis of e-Commerce Customer Management System Based on Mobile Edge Computing
}

\author{
Wenxing Chen $\mathbb{D i D}^{1}$ and Bin Yang $\mathbb{D}^{2}$ \\ ${ }^{1}$ Henan Quality Polytechnic, Pingdingshan 467000, China \\ ${ }^{2}$ Henan University of Urban Construction, Pingdingshan 467000, China \\ Correspondence should be addressed to Wenxing Chen; sun20211120@163.com and Bin Yang; 20210001@hncj.edu.cn
}

Received 22 November 2021; Revised 8 December 2021; Accepted 16 December 2021; Published 3 January 2022

Academic Editor: Le Sun

Copyright (c) 2022 Wenxing Chen and Bin Yang. This is an open access article distributed under the Creative Commons Attribution License, which permits unrestricted use, distribution, and reproduction in any medium, provided the original work is properly cited.

\begin{abstract}
Energy efficiency optimization of mobile edge computing e-commerce clients and reasonable management of server computing resources are worth further study. The participant of the algorithm game model proposed in this paper is mobile e-commerce customer management. The decision space is a two-dimensional space composed of unloading decision and power control, and the benefit function is the energy efficiency function and delay function. The existence and uniqueness of the multidimensional game model are proved theoretically. The simulation results show that the proposed multidimensional game based energy efficiency optimization algorithm of mobile edge computing can reduce the energy consumption and delay of mobile terminals and improve the energy efficiency of unloading calculation under the same task compared with the game scheme without considering power consumption control when the number of e-commerce customer management is larger. This paper deduces the optimal load migration decision of mobile e-commerce customer management and the optimal pricing strategy of mobile edge cloud service providers and proves that the optimal decision and optimal pricing constitute the Starkberg equilibrium. The semidistributed and decentralized task transfer decision-making mechanisms are designed, respectively, and the management decision-making behaviors of mobile e-commerce customers in the mobile edge cloud energy trading market are studied by numerical analysis, as well as the time efficiency of the two mechanisms.
\end{abstract}

\section{Introduction}

Mobile edge is defined as the technology by the European Telecommunications Standards Institute (ETSI), providing service environments, within wireless access networks and in the vicinity of mobile users [1]. ETSI on mobile edge computing is considered to be an important emerging technology and an important part of the next generation of networks.

The video stream from the monitoring device is processed and analyzed on the MEC server, and the meaningful data is extracted from the video stream. You can transfer valuable data that are inherently cooperative in data, edge computing, and data transfer in the prediction link. Data processing can be performed on a wooden MEC server rather than a centralized server to provide a good user experience. The Internet of Things generates additional messages over telecommunications networks, requiring gateways to aggregate messages and ensure low latency and security. We introduce a new architecture for collecting $[2,3]$, classifying, and analyzing IoT data flows using MEC servers that manage various protocols, message distribution, and analytical processing. A vibrant ecosystem creates new opportunities for mobile operators, applications, and content providers.

With the rapid development of e-commerce in today's society, customers also have a great impact on the development of e-commerce enterprises. Firstly, in the e-commerce environment, customers can browse, understand, and purchase products and services through the network; through realization, such as customer purchasing power, and customer loyalty; then through network communication and other ways to timely contact customers; and through care and other marketing means to obtain more customer 
support. The development of e-commerce enterprises needs the support of an e-commerce customer relationship management system, which provides a scientific basis for enterprises to make scientific decisions and market development strategies.

\section{Related Work}

In e-commerce customer relationship management systems, data mining technology is integrated to realize the analysis of existing customer data, establish an analysis model for customers, analyze customers through the data model, classify customers, and find more potential customers $[4,5]$. The e-commerce customer relationship management system in the domestic market system is not perfect but also made some achievements. Earlier construction of e-commerce customer relationship management systems includes telecom and mobile companies, the banking industry, and aviation. Customer relationship management not only includes the addition, deletion, and modification of customer information but also includes the analysis of customer data, understanding customers' hobbies, timely finding which customers are easy to lose, and vigorously digging potential customer markets. Domestic theoretical research in these aspects has achieved initial results, such as the use of the genetic algorithm, artificial neural network, and other classification acquisition methods for customer data analysis and prediction. The system structure of domestic e-commerce customer relationship management system is relatively simple, the function is relatively simple, and most of the management systems are only to achieve the management of customer basic information data. Compared with foreign countries, the development and research of China's e-commerce customer relationship management system are still relatively backward and single $[6,7]$. All in all, abroad and at home, now the common development of the world economy is not a good customer relationship management system support; it is not a successful future business, helps the customer management and analysis, and enhances the working efficiency of the enterprise; the Lord will help improve the position and role of enterprise in the market.

Among them, the energy efficiency optimization of mobile edge computing e-commerce customer relationship management system includes a well-designed task unloading scheme, communication channel allocation, and transmission power control. In literature [8], an effective MEC system calculation model is proposed to combine computing and communication cooperation to optimize system energy efficiency. In literature [9], dynamic voltage scaling technology is used to jointly optimize the computing speed, transmission power, and unloading ratio of mobile devices to improve energy efficiency. In literature [10], transmission power control and unloading decision of MEC systems under single user conditions are studied. As an improvement, the authors of literature [11] extended the conclusions in literature to multiuser MEC systems, and combined with resource allocation, they optimized the energy efficiency of multiuser MEC systems through Lyapunov. In [12], an integrated framework for computational unloading is proposed, which takes into account unloading decisions, to optimize energy efficiency. However, in literature [13], intercustomer interference of e-commerce is not taken into account when the unloading decision of mobile users is determined. Studies show that effective power control can effectively suppress intercustomer interference of e-commerce in a small e-commerce customer network [5, 14]. Therefore, some MEC algorithms based on transmission power control are proposed. For example, the transmission power control algorithms proposed in the literature $[15,16]$ and the algorithms proposed in the literature [17] are effective in multiuser MEC systems, but they are centralized and not always effective in distributed systems. As the computing resources of the MEC server are limited, more and more terminal devices will connect to the server in the future when the number of mobile devices is increasing exponentially. Therefore, how to reasonably manage computing resources has become a significant research direction. Literature [18] proposed a branch and bound algorithm (VF-BNB) based on variable fusion to optimize computing resources. The model in literature [19] is a mobile edge computing system with a large number of low-power mobile terminals. Therefore, reasonable allocation of computing resources is the key to achieving system performance. The problem of computing resource allocation in the upstream MEC system under the minimum to maximum fairness criterion is studied. Literature [20] uses the Lagrange multiplier method with a predetermined resource allocation strategy to solve the problem of computing resource allocation. The above literature conducts quantitative analysis from the perspective of limited computing resources and then completes resource management through carefully designed algorithms but ignores the mobility of terminal devices. If the user continues to move, the original request discharge calculation of the server may not be able to timely send the result in the user's next position. User mobility prediction distribution and which server to use are studied in the literature [21]. Literature [22] studies a kind of virtual machine migration strategy based on the Markov decision process; in the literature [23], congestion in the network virtual machine migration strategy is studied.

However, the commonly used assumptions in the above algorithms for energy efficiency optimization of e-commerce CRM systems based on MEC mobile edge computing have certain limitations: the computing resources of MEC servers are limited, and unloading a large number of tasks will bring considerable queuing delay. In the scenario of ultradense networks (UDN), interference between e-commerce customers can significantly affect data rates and lead to unexpected transmission delays, which can lead to low energy efficiency and long delays. Therefore, interference and queuing problems cannot be ignored in reality. Although MEC has many benefits, the energy efficiency of its mobile terminal cannot be ignored. Game theory is a powerful distributed mechanism design tool. In order to maintain the balance of the system and consider the interests of each user, it is of great significance to use game theory as a tool to study energy efficiency in the MEC system. In the MEC system, virtual machine migration is usually adopted for user 
movement, but for some applications with high instantness requirements and small task volume, the migration cost is too large. Although mobility prediction is carried out, the model is an ideal case of uniform motion. In general, the user's movement has acceleration, so there is room for further study of this scheme.

\section{Mobile Edge Computing for e-Commerce Customer Management Energy Efficiency Optimization Framework and Key Technology Analysis}

3.1. System Architecture. Enabling MEC applications to be realized as pure software entities running on MEC hosts, mobile edge platforms provide the base needed to run applications. MEC applications are run as virtual machine VMS on top of a virtualized infrastructure that includes a data platform that enforces traffic rules received by mobile edge platforms and routes traffic between the application forest network and the external network. In host level management including mobile platform edge and virtualization infrastructure, the former is responsible for the management of electronic commerce application of life cycle and rule requirements, including e-commerce customer management system business license, flow rule, and the domain name system conflict resolution, which is responsible for the allocation, management, and releasing the visualization of virtualization infrastructure (computing, storage, and network resources). The architecture diagram is shown in Figure 1.

As shown in Figure 2, the framework of the e-commerce customer management system of edge computing is divided into mobile edge computing level, mobile edge e-commerce customer system host level, and network-level entity. The host layer of the mobile edge e-commerce customer system is the basic part of the framework, which consists of mobile edge host and host management. Virtualization infrastructure can provide ME with the application with computing, storage, and network resources and can provide ME with the application with persistent storage and access to relevant information; it includes a data forwarding plane, used to receive from ME platform data forwarding rules, as well as between each application, service, and network flows. The ME platform receives traffic forwarding rules from the ME platform manager, ME applications, or ME services and sends instructions to the forwarding plane based on the forwarding rules. In addition, the ME platform supports the configuration of local domain name system (DNS) proxy servers to redirect data traffic to corresponding applications and services. The ME platform can also communicate with other ME platforms through Mp3 reference points, which can serve as the basis for interconnecting different $\mathrm{ME}$ platforms in the collaboration mechanism of distributed MEC systems.

Among them, intelligent service is based on the modeldriven unified service framework, which realizes the coordination of development and deployment intelligence through the development service framework and deployment operation service framework, and can realize the consistency of software development and deployment operation automation. Intelligent business orchestration defines end-to-end business flows through business support capabilities to achieve business agility. Connected computing achieves a minimalist architecture, shielding services from the complexity of edge intelligent distributed architecture, realizing automation and visualization of operation, information and communication technology infrastructure deployment and operation, and supporting intelligent collaboration between edge computing resource services and industry business needs. Intelligent Edge computing node $(\mathrm{ECN})$ is compatible with a variety of heterogeneous connections, supports real-time processing and response, and provides hardware and software integration security. The three-tier model is mainly for scenarios where services are deployed to one or more scattered regions, and the business flow in each region is small, such as smart street lamps, smart elevators, and smart environmental protection. After the intelligent asset is processed locally, multiple types of service data are aggregated to the intelligent gateway along the north-south direction. The intelligent gateway processes real-time service requirements locally and aggregates nonreal-time data and sends it to the cloud for processing.

3.2. Research on Key Technology of Energy Efficiency Optimization. An important part of calculating unload is deciding whether to unload, whether all or part of it applies, what to unload, and how to unload. The uninstall decision depends on the application model categorized according to three criteria. In the task execution schedule, we need to know how to select the task execution sequence to reduce the task execution time, and so on. For optimization problems with equality and inequality constraints, the general form is

$$
\min h(x), f_{i}(x) \leq 0, \quad i=1, \ldots, n
$$

In the optimization problem-solving algorithm, the KKT condition is the necessary and sufficient condition for the convex optimization problem to obtain the optimal solution, and the KKT condition is

$$
\delta h(x)+\sum_{i=1}^{n} u_{i} f_{i}(x)+\sum_{j} v_{j}=0 .
$$

The in-memory data grid technology is adopted, and improvements are made in storing lightweight and highperformance access, forming a lightweight and high-performance edge service catalog. On this basis, considering the influence of the mobile edge computing environment and the dynamic change of directory nodes, the synchronization and consistency guarantee technology of the edge service directory is studied. The core methods to solve this problem include the following three aspects: (1) taking into account the synchronization modes such as upstream and downstream synchronization and peer synchronization, the problem of information service sharing can be solved by flexibly selecting suitable directory synchronization mode, 

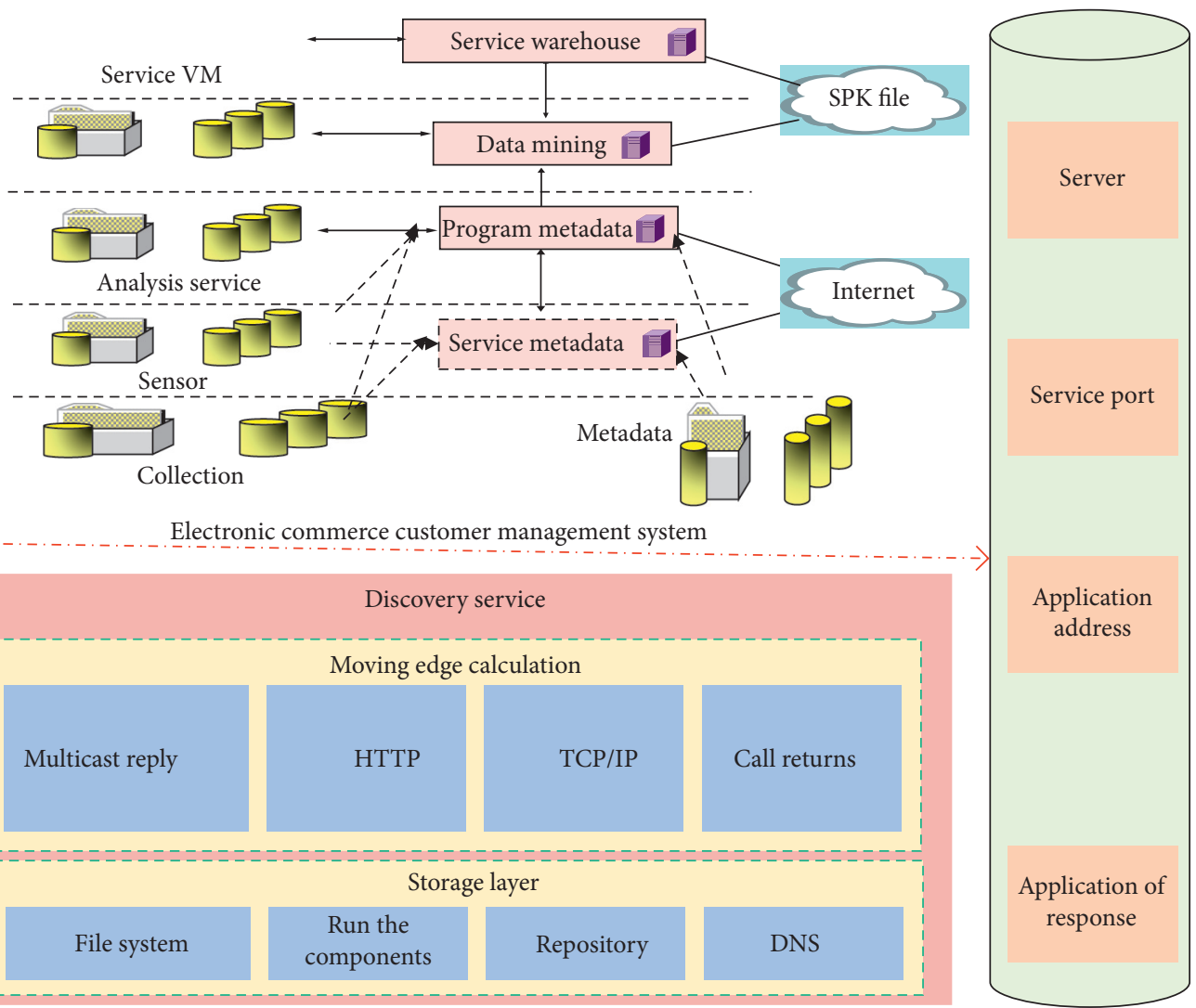

Figure 1: Optimized e-commerce customer management architecture for mobile edge computing energy efficiency.
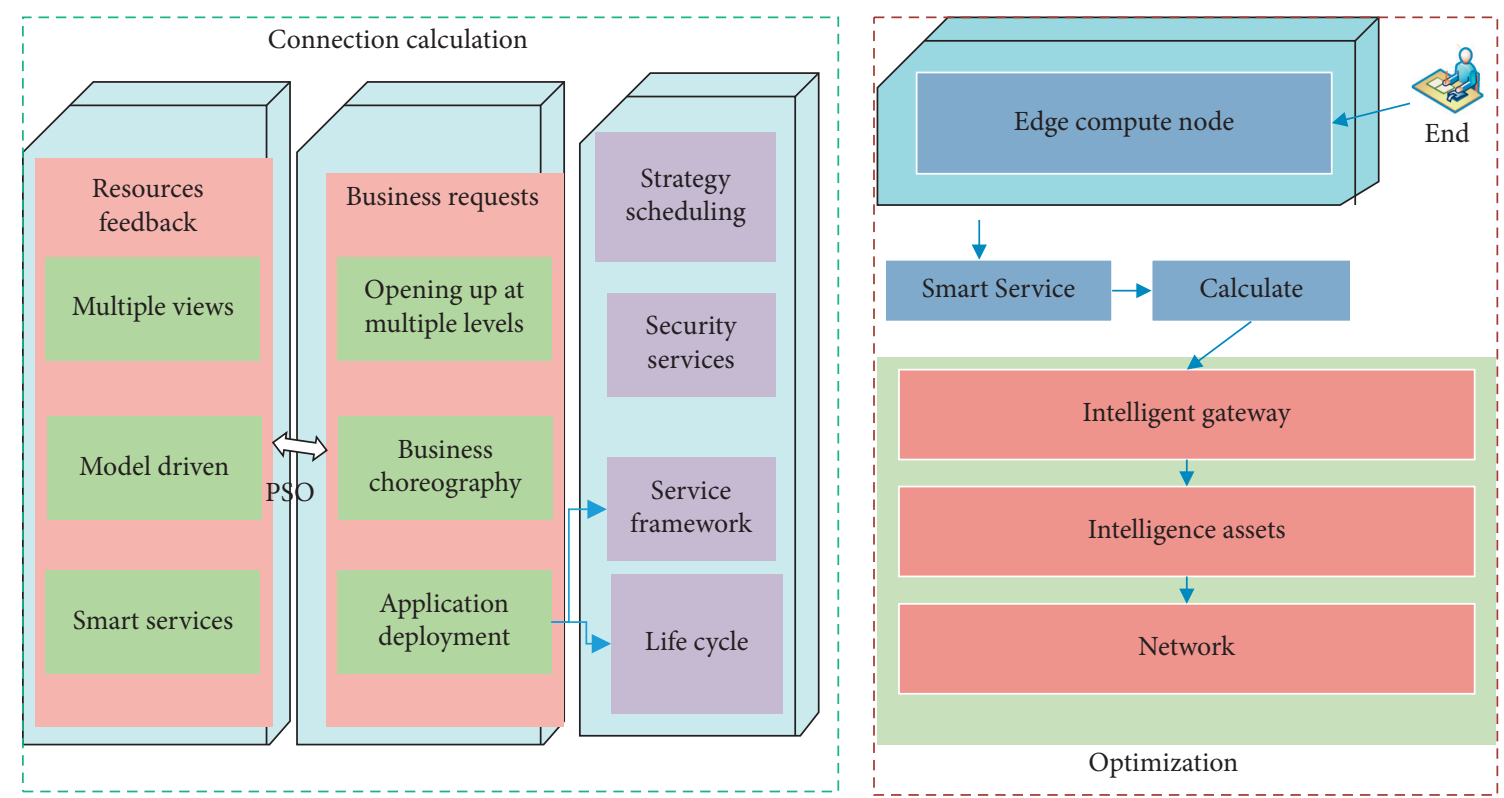

FIGURE 2: Energy efficiency optimization architecture of mobile edge computing for e-commerce customer management.

supporting dynamic discovery, flexible networking, and service sharing among nodes, and realizing the dynamic collaborative operation of service catalog; (2) we adopt the updating mechanism of active monitoring to ensure that the service information recorded in the decentralized edge service catalog is consistent, real-time, and effective and improve the availability of services built on the distributed structure; (3) a new distributed consistency algorithm based on state machine replication is proposed. By sorting and executing the uncoupling consensus protocol and the operation commands, the operation commands can be concurrently submitted for all backups at any time without 
determining the order of the operation commands and can be executed in the same order on all backups. The structure of the service catalog can be divided into three layers, namely, catalog summary layer, catalog middle layer, and service instance layer. The aggregation layer of the service catalog and the directory area of the middle layer have a central service catalog sharing model, which is mainly used in the service catalog. The directory services use the directory equivalent middle layer region sharing service model. The mobile edge computing service catalog model architecture is shown in Figure 3.

The existing microservice system mainly adopts a rulebased routing policy to realize the routing function of the service based on the service catalog information provided by the service registry and the service status information reported by the service agent. However, the mobile edge computing environment is complex and changeable and has significant dynamic characteristics. If a simple and rulesbased routing strategy is directly adopted, the system performance cannot be optimized. Therefore, how to select the service invocation path intelligently according to the service quality, service invocation history, dynamic use of system resources, and other information in the current application environment, as well as the historical service routing strategy, is the key problem of edge service routing address.

\section{Energy Efficiency Optimization Algorithm for e-Commerce Customer Management Based on Mobile Edge Computing}

In the two-dimensional space composed of decision-making, interference between users can be reduced through the reasonable design of transmitting power size, the upper limit of MEC server computing resources can be set, queuing delay of the mobile terminal is added, optimization target is energy consumption and delay of the mobile terminal, and game model is established. The core idea is to select the most suitable unloading decision and transmitting power for each user under the current situation through the game, so as to reduce interference so that more users can have the opportunity of unloading computing and improve the energy efficiency of the mobile terminal. The purpose of using the game algorithm is to select the most suitable decision so as to optimize the energy consumption and delay function:

$$
\min _{i} f_{i}\left(s_{i n},-s_{i n}\right) .
$$

This algorithm is a multiuser system, which uses parallel time slots, and each user calculates its own optimal response decision in each time slot. During initialization, the user's decision is to uninstall the MEC server for calculation, and the upload power is randomly selected. In each time slot, the user collects energy efficiency information: other e-commerce customers need to uninstall the MEC server computing the number of users, their choice of channel, and according to the information transmission rate calculation, each channel interference, and the total number of users, in MEC server to determine whether the user needs to line up. From these parameters, the optimal response, namely, the unload decision (whether to unload) and the upload power size, can be calculated. In the process of inference in means-purpose with an online autonomous learning algorithm to realize the optimal strategy of child intent, intention to achieve group, and the agent need to consider its own state-action value function updates for other agent update value functions can be without a second thought, thus greatly reducing the space complexity of the algorithm and improving the learning speed. The algorithm flow is shown in Figure 4.

Customer Relationship Management (CRM) system is a business strategy of an enterprise. It divides and effectively organizes enterprise resources according to customer conditions and cultivates customer-centered operation behavior and customer-centered business process, so as to improve enterprise profitability, increase revenue, and improve customer satisfaction. The development of e-commerce requires enterprises to transform all internal and external business processing mechanisms into "customer satisfaction" service subjects and use new measures and technologies to reduce internal consumption and cost so that the business processing capacity of enterprises constantly meets the needs of Internet customers' rapid operation. One of the purposes of establishing a customer relationship management system is to improve the quality of customer service, depending on the customer service management module, which can effectively achieve customer service and improve customer trust in enterprises and products.

The function module has a customer service console, customer service event record, customer complaint management, and consultation library. The customer service console provides quick inquiries for customer service personnel and provides assistance for customer service personnel. For example, customer service personnel can quickly check the handling of complaints through this module, timely contact customers to solve the unresolved complaints, or carry out customer care and return visits for customers classified as easy to lose, so as to reestablish the trust between customers and enterprises. According to the optimal pricing strategy of customer and demand, the mobile edge energy efficiency mechanism is designed by semidistributed method and decentralized method respectively. See Table 1.

\section{Example Verification}

This part analyzes the behavior of mobile users with five user scenarios. These five users are named U1, U2, U3, U4, and $\mathrm{U} 5$, respectively, and the number of CPU cycles for each user task is set. The task is sorted in descending order of energy efficiency. Figure 5 shows the trend of users' load migration decisions as energy efficiency pricing strategy changes. When energy efficiency pricing is low, all users migrate their load tasks to energy efficiency for processing. After that, as energy efficiency pricing increases, users will gradually reduce the proportion of tasks migrating to energy efficiency. When energy efficiency pricing reaches a certain level, the lower bound of optimal energy efficiency pricing, user U5 will begin to reduce its load migration ratio. As energy efficiency pricing increases further, other users will respond 


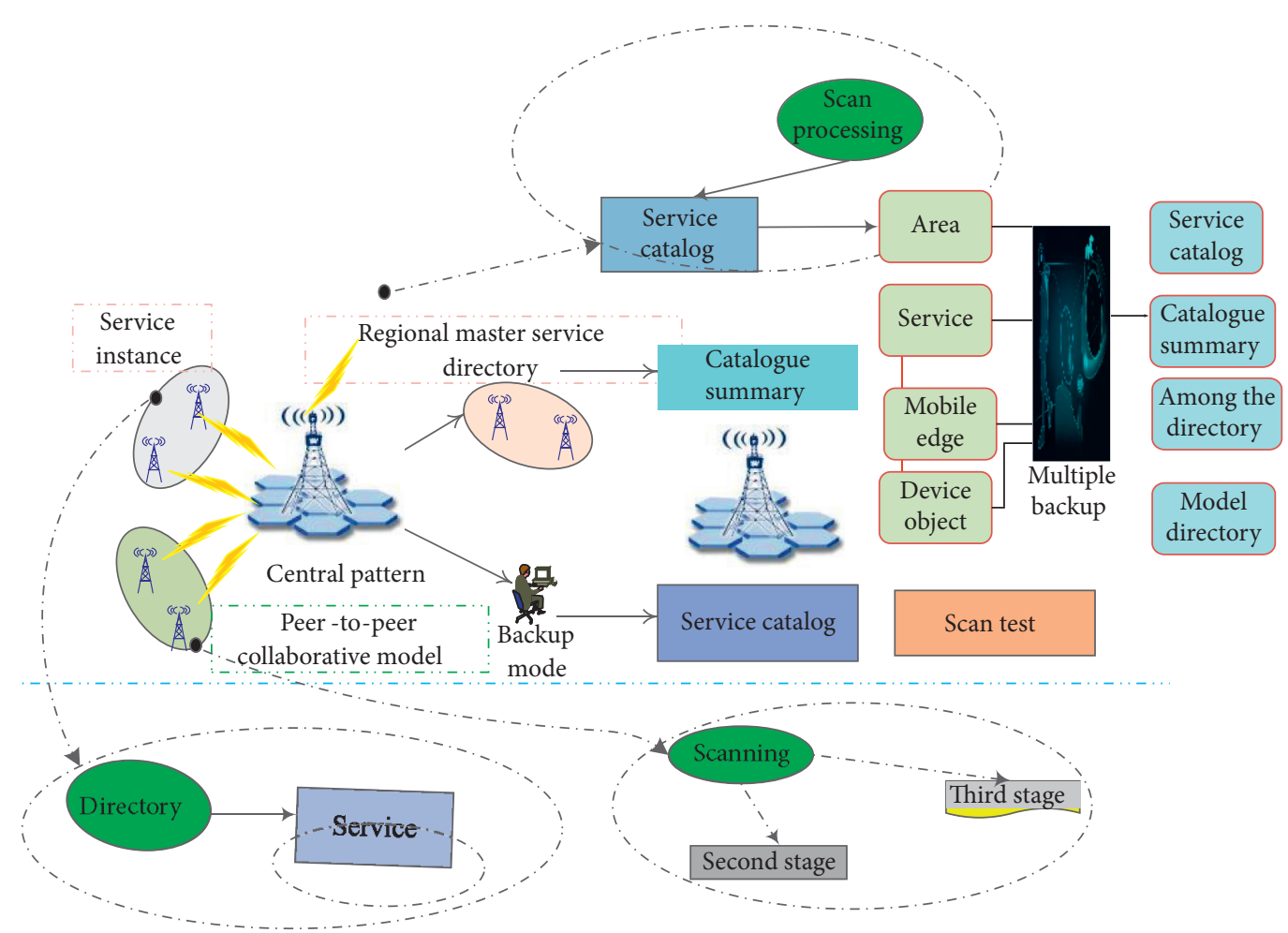

FIgURE 3: Mobile edge computing service catalog model architecture.

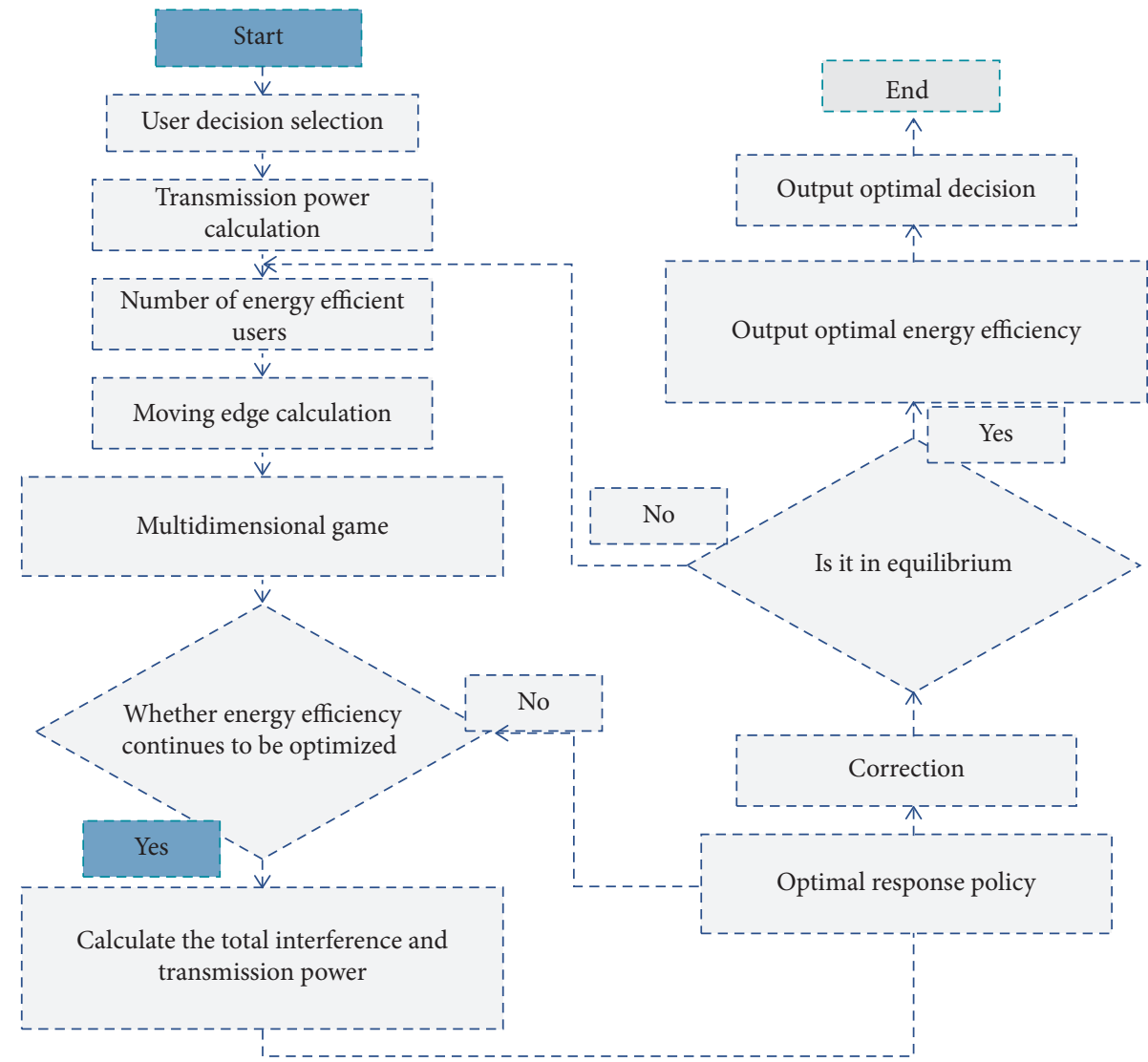

FIGURE 4: Flow chart of energy efficiency optimization algorithm for moving edge computing based on a multidimensional game. 
TABLE 1: Design of energy efficiency mechanism of moving edge by the decentralized method.

On each mobile user's side

1: if $E_{i} \geq 0$, then

2: user I sends the load migration information to the customer

3: waiting for price $P$

4: end if

5: each user calculates the optimal load migration policy based on the received customer demand $P$

6: each user loads some tasks to energy efficiency $f$ and locally calculates the remaining tasks on the demand side

7: waiting for user information

8: arrange all user information in descending order of energy efficiency $f$

9: calculate $P$ and broadcast it to all users

10: wait for the user load migration task to be executed

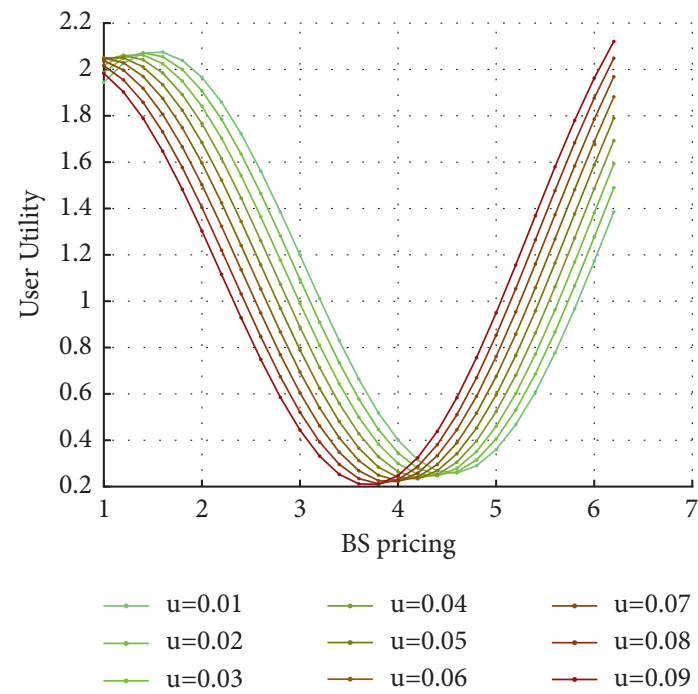

Figure 5: Decision analysis of energy efficiency change of ecommerce management at mobile user edge.

to the price and reduce the proportion of load transfer tasks. When the price of energy efficiency reaches a high level, the upper bound of optimal pricing strategy for energy efficiency), no user migrates tasks to energy efficiency for processing. Obviously, when energy efficiency pricing is lower than or higher than the lower bound of its optimal pricing strategy, energy efficiency cannot obtain optimal utility benefits. At the same time, in the game of energyefficient computing resources among users, users with higher energy efficiency, that is, high efficiency of task transfer, have a stronger tendency of task transfer.

For all users, the computing power of load migration is evenly distributed in the interval. For the decentralized algorithm, its operating efficiency is related to the initial pricing strategy set by the algorithm. When evaluating the efficiency of the algorithm, the average number of iterations under the two conditions is taken as the number of iterations of the algorithm. The time efficiency of the two algorithms is shown in Figure 6. The solid blue line represents the algorithm time of this distributed algorithm, and the dotted red line represents the number of iterations of the decentralized algorithm. For the decentralized algorithm, the number of iterations of the algorithm is independent of the number of users participating in the game because the algorithm is mainly searching the feasible region of the pricing

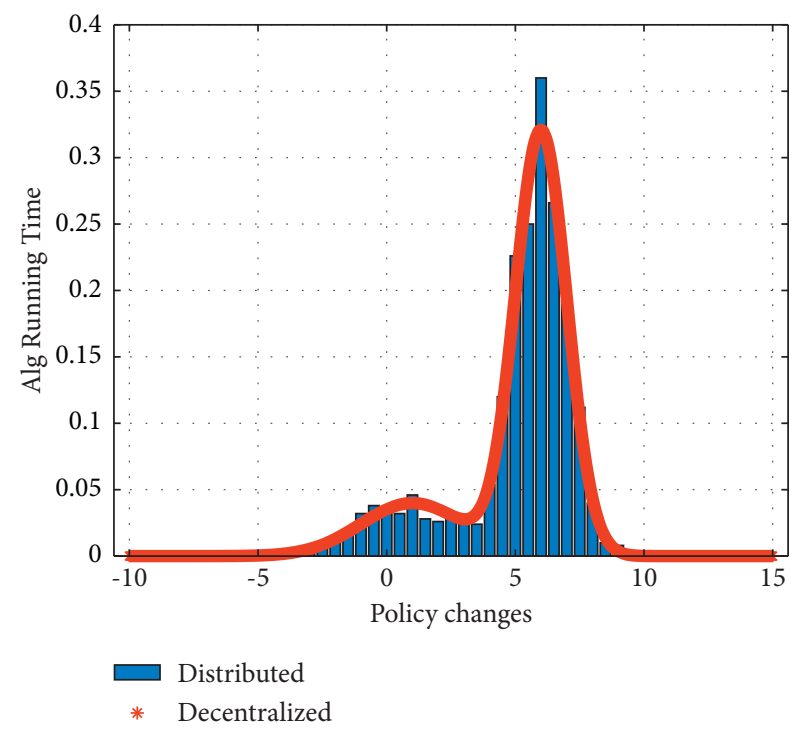

Figure 6: Time efficiency of mobile edge cloud energy consumption optimization algorithm.

strategy. For the semidistributed algorithm, the relationship between the algorithm complexity and the number of users is superlinear, which also leads to the increase of the number of users participating in the game; the algorithm strategy will produce a certain amount of algorithmic calculation pressure on the base station.

As shown in Figure 7, when the initial speed is $V=60 \mathrm{~km} / \mathrm{h}$ and $a=1.5 \mathrm{~m} / \mathrm{s}^{2}$, the success rate increases with the increase in the number of MEC servers, because the increase in the number of servers means that the number of available servers is increasing. As the speed increases, the user moves more distance, and the MEC server has a limited range of connections; the success rate decreases as the number of tasks increases. Moreover, with the expansion of the mobile range, it can be seen that the success rate of CRMAEKF is better than PA.

Figure 8 shows the comparison of total system energy consumption and delay of the two algorithms, namely, the sum of all users and the comprehensive consideration of energy consumption and delay, so the unit is not considered. The higher the energy consumption and delay are, the lower the energy efficiency is. As shown in Figure 8, as the number of users increases, both MQO and ODPC show an increasing trend, but MQO has lower energy consumption and delay 

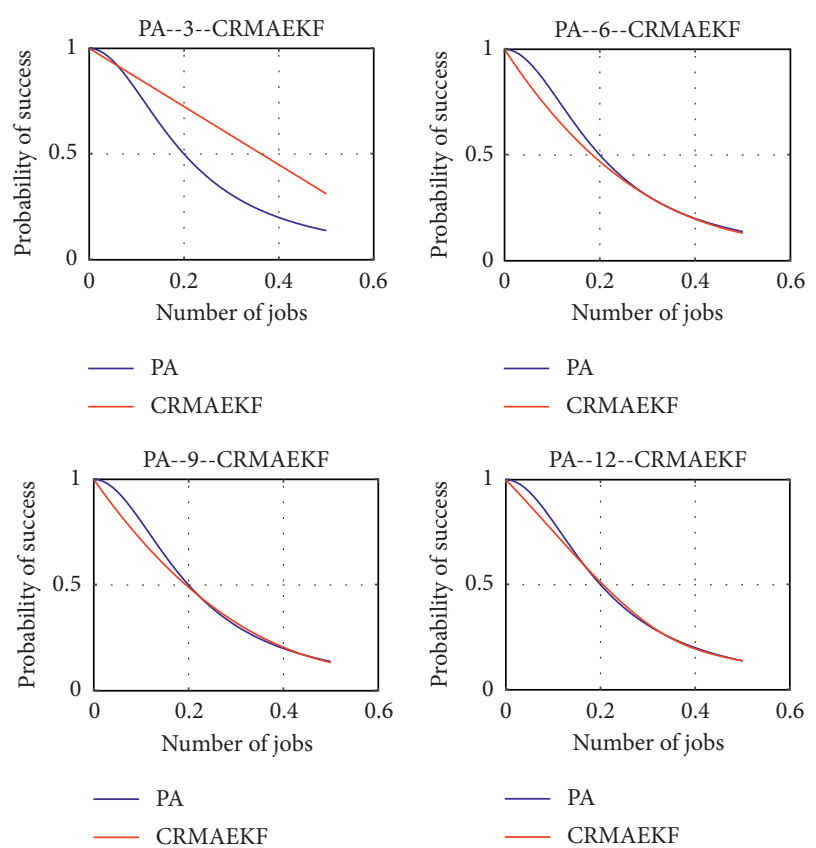

Figure 7: Success rate of different tasks at initial velocity $V=60 \mathrm{~km} / \mathrm{h}$ and $A=1.5 \mathrm{~m} / \mathrm{s}^{2}$.

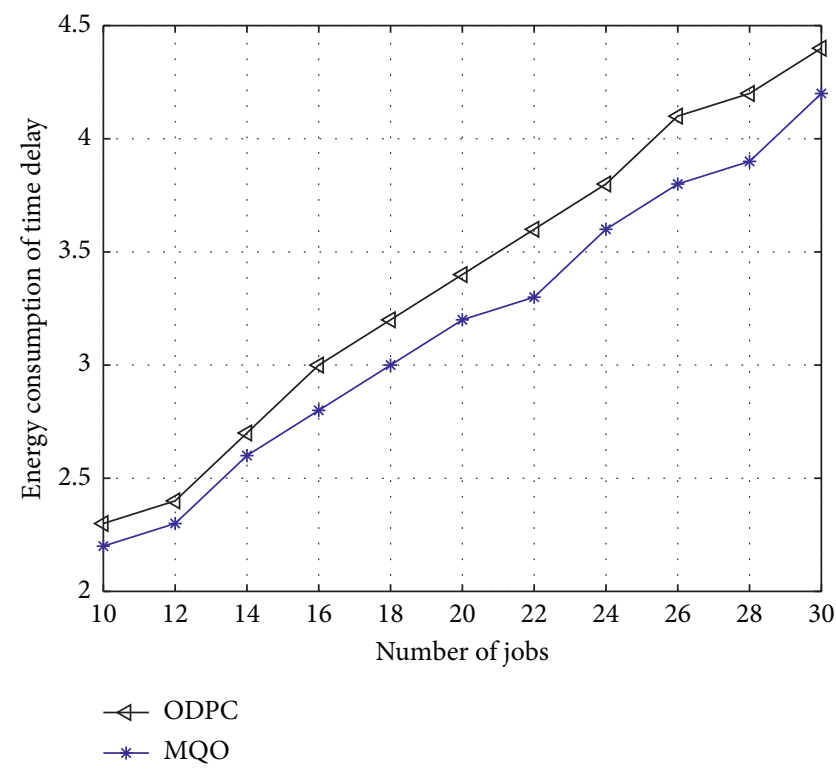

FIGURE 8: User energy consumption and delay of different numbers of e-commerce customers.

than ODPC. As the number of e-commerce customers increases, so does the number of users. As more and more users choose to unload computing, the queuing delay and interference increase significantly. Under the control of unloading quantity, the energy consumption and delay of MQO are lower than those of ODPC, which proves that the optimization of energy consumption and delay of MQO is superior to that of ODPC.

This paper studies the energy efficiency optimization of the mobile terminal using a game in a multiuser system and proposes an energy efficiency optimization algorithm of mobile edge computing based on multidimensional game. The decision of the game model is a two-dimensional space, which consists of an unloading decision and power control. MEC server computing resources are limited. If the MEC server is overloaded, queuing delay will be introduced. The existence and uniqueness of Nash equilibrium are proved by theoretical deduction. According to this algorithm, the energy consumption and delay of the mobile terminal can be reduced, and the smaller the energy consumption is, the higher the energy efficiency is. And because transmitting power is considered jointly in the decision space, the number of users in unloading calculation can be increased by controlling transmitting power to reduce interference.

\section{Conclusion}

This paper proposes an energy efficiency optimization algorithm of mobile edge computing based on multidimensional game. Aiming at the energy efficiency optimization problem of the mobile terminal in the multiuser system, this paper aims to improve the energy efficiency of the mobile terminal, establish a communication model and calculation model, set upper limit for MEC server computing resources, and add queuing delay. The multidimensional game model is constructed based on game theory, in which the game decision is a two-dimensional space composed of an unloading decision and power control. It is proved that the Nash equilibrium of this multidimensional game model exists and is unique through theoretical deduction, and the Nash equilibrium solution is finally obtained. The simulation experiment proves that the proposed energy efficiency optimization algorithm of mobile edge computing based on multidimensional game can improve the energy efficiency of mobile terminal compared with the one-dimensional game scheme without power control and reduce interference by controlling transmission power so that more users can unload tasks to MEC server for computing processing. The development of e-commerce and the management of customer relationships should be rationally and effectively combined. The development of e-commerce has promoted the research of customer management and improved the importance of customer relationships. The improvement of customer relationships is helpful for e-commerce enterprises to seize the high-end market, expand business development, and make scientific development strategies. The two complement each other.

\section{Data Availability}

The data used to support the findings of this study are available from the corresponding author upon request.

\section{Conflicts of Interest}

The authors declare that they have no conflicts of interest.

\section{Acknowledgments}

This study was sponsored by HeNan Quality Polytechnic. 


\section{References}

[1] Y. Yang, Z. Mei, and B. Zheng, "Design of enterprise management system based on edge computing architecture," Mobile Information Systems, vol. 2021, no. 10, 12 pages, Article ID 5512958, 2021.

[2] Z. Zheng and X. Zhou, "Design and simulation of ship energy efficiency management system based on data analysis," Journal of Coastal Research, vol. 94, no. sp1, pp. 552-567, 2019.

[3] R. Gopi, S. T. Suganthi, R. Rajadevi, P. Johnpaul, N. Bacanin, and S. Kannimuthu, "An enhanced green cloud based queue management (GCQM) system to optimize energy consumption in mobile edge computing," Wireless Personal Communications, vol. 117, no. 4, pp. 3397-3419, 2021.

[4] J. Sun, "Research on resource allocation of vocal music teaching system based on mobile edge computing," Computer Communications, vol. 160, no. 2, pp. 345-365, 2020.

[5] H. Sun, F. Zhou, and R. Q. Hu, "Joint offloading and computation energy efficiency maximization in a mobile edge computing system," IEEE Transactions on Vehicular Technology, vol. 68, no. 3, pp. 3052-3056, 2019.

[6] T. Wang, G. Zhang, and X. Yang, "A trusted and energy efficient approach for cluster-based wireless sensor networks," International Journal of Distributed Sensor Networks, vol. 4, pp. 131-143, 2016.

[7] T. Le Tan, "Successful factors of implementation electronic customer relationship management (e-CRM) on E-commerce company," American Journal of Software Engineering and Applications, vol. 6, no. 5, pp. 121-127, 2017.

[8] Z. Di, Z. Zhenyu, and Z. Zhengyu, "Energy efficiency analysis of ICN assisted 5G IoT system," Wireless Communications and Mobile Computing, vol. 2017, Article ID 6579467, 9 pages, 2017.

[9] P. Wumin and M. Rui, "Research on improvement of China mobile customer relationship management system based on apriori algorithm," Boletin Tecnico/Technical Bulletin, vol. 55, no. 19, pp. 520-526, 2017.

[10] W. Sun and Y. Gao, "The design of university physical education management framework based on edge computing and data analysis," Wireless Communications and Mobile Computing, vol. 2021, no. 2, 8 pages, Article ID 5537471, 2021.

[11] S. Azeem, N. Nasir, and S. Kousar, "Impact of E-commerce investment and enterprise performance based on customer relationship management," International Journal of Psychosocial Rehabilitation, vol. 24, no. 9, pp. 9-31, 2020.

[12] H. Jiang, Y. Wang, and X. Yue, "Performance analysis of NOMA-based mobile edge computing with imperfect CSI," EURASIP Journal on Wireless Communications and Networking, vol. 2020, no. 1, 138 pages, 2020.

[13] S. Zhou, W. Jadoon, and J. Shuja, "Machine learning-based offloading strategy for lightweight user mobile edge computing tasks," Complexity, vol. 2021, no. 3, 11 pages, Article ID 6455617, 2021.

[14] Y. Sun, C. Song, S. Yu, Y. Liu, H. Pan, and P. Zeng, "Energyefficient task offloading based on differential evolution in edge computing system with energy harvesting," IEEE Access, vol. 9, Article ID 16383, 2021.

[15] P. Corcoran, "Mobile-edge computing and Internet of Things for consumers: Part II: energy efficiency, connectivity, and economic development," IEEE Consumer Electronics Magazine, vol. 6, no. 1, pp. 51-52, 2016.

[16] M. Xia, Z. Huang, L. Tian et al., "SparkNoC: an energy-efficiency FPGA-based accelerator using optimized lightweight
CNN for edge computing," Journal of Systems Architecture, vol. 115, no. 4, Article ID 101991, 2021.

[17] S. Han, X. Xu, S. Fang et al., "Energy efficient secure computation offloading in NOMA-based mMTC networks for IoT," IEEE Internet of Things Journal, vol. 6, no. 3, pp. 5674-5690, 2019.

[18] Q. Wang, S. Guo, J. Liu, and Y. Yang, "Energy-efficient computation offloading and resource allocation for delaysensitive mobile edge computing," Sustainable Computing: Informatics and Systems, vol. 21, no. MAR., pp. 154-164, 2019.

[19] X. Liu, J. Yang, and C. Zou, "Collaborative edge computing with FPGA-based CNN accelerators for energy-efficient and time-aware face tracking system," IEEE Transactions on Computational Social Systems, vol. 4, no. 9, pp. 12-27, 2021.

[20] D. Zhang, C. Gong, and T. Zhang, "A new algorithm of clustering AODV based on edge computing strategy in IOV," Wireless Networks, vol. 27, no. 4, pp. 13-31, 2021.

[21] R. Tatiara, A. N. Fa Jar, and B. Siregar, "Analysis of factors that inhibiting implementation of information security management system (ISMS) based on ISO 27001," Journal of Physics Conference, vol. 978, Article ID 12039, 2017.

[22] Y. Bian, M. Hu, Y. Wang, and H. Xu, "Energy efficiency analysis of the economic system in China during 1986-2012: a parallel slacks-based measure approach," Renewable and Sustainable Energy Reviews, vol. 55, pp. 990-998, 2016.

[23] S. Z. Zhao and L. Zhang, "Analysis of enterprise management information system based on cloud computing," Journal of Physics: Conference Series, vol. 1533, Article ID 22038, 2020. 